\title{
Clustering of adherence to personalised dietary recommendations and changes in healthy eating index within the Food $4 M e$ study
}

Katherine M Livingstone ${ }^{1}$, Carlos Celis-Morales ${ }^{1}$, Jose Lara' ${ }^{1}$ Clara Woolhead ${ }^{2}$, Clare B O'Donovan ${ }^{2}$, Hannah Forster ${ }^{2}$, Cyril FM Marsaux ${ }^{3}$, Anna L Macready ${ }^{4}$, Rosalind Fallaize ${ }^{4}$, Santiago Navas-Carretero ${ }^{5,6}$, Rodrigo San-Cristobal ${ }^{6}$, Silvia Kolossa ${ }^{7}$, Lydia Tsirigoti ${ }^{8}$, Christina P Lambrinou ${ }^{8}$, George Moschonis ${ }^{8}$, Agnieszka Surwiłto9, Christian A Drevon ${ }^{10}$, Yannis Manios ${ }^{8}$, Iwona Traczyk ${ }^{9}$, Eileen R Gibney ${ }^{2}$, Lorraine Brennan ${ }^{2}$, Marianne C Walsh ${ }^{2}$, Julie A Lovegrove ${ }^{4}$, J Alfredo Martinez ${ }^{6}$, Wim HM Saris ${ }^{3}$, Hannelore Daniel7, Mike Gibney ${ }^{2}$ and John C Mathers ${ }^{1, *}$ on behalf of the Food4Me Study 'Human Nutrition Research Centre, Institute of Cellular Medicine, Newcastle University, Biomedical Research Building, Campus for Ageing and Vitality, Newcastle upon Tyne NE4 5PL, UK: ${ }^{2}$ UCD Institute of Food and Health, University College Dublin, Dublin, Republic of Ireland: ${ }^{3}$ Department of Human Biology, NUTRIM School of Nutrition and Translational Research in Metabolism, Maastricht University Medical Centre, Maastricht, The Netherlands: ${ }^{4}$ Hugh Sinclair Unit of Human Nutrition and Institute for Cardiovascular and Metabolic Research, University of Reading, Reading, UK: ${ }^{5}$ Center for Nutrition Research, University of Navarra, Pamplona, Spain: ${ }^{6}$ CIBER Fisiopatología Obesidad y Nutrición (CIBERobn), Instituto de Salud Carlos III, Madrid, Spain: ${ }^{7}$ ZIEL Research Center of Nutrition and Food Sciences, Biochemistry Unit, Technical University of Munich, Munich, Germany: ${ }^{8}$ Department of Nutrition and Dietetics, Harokopio University, Athens, Greece: ${ }^{9}$ National Food \& Nutrition Institute (IZZ), Warsaw, Poland:

${ }^{10}$ Department of Nutrition, Institute of Basic Medical Sciences, Faculty of Medicine, University of Oslo, Oslo, Norway

Submitted 20 January 2016: Final revision received 18 May 2016: Accepted 20 June 2016: First published online 8 August 2016

\begin{abstract}
Objective: To characterise clusters of individuals based on adherence to dietary recommendations and to determine whether changes in Healthy Eating Index (HEI) scores in response to a personalised nutrition (PN) intervention varied between clusters.

Design: Food4Me study participants were clustered according to whether their baseline dietary intakes met European dietary recommendations. Changes in HEI scores between baseline and month 6 were compared between clusters and stratified by whether individuals received generalised or PN advice.

Setting: Pan-European, Internet-based, 6-month randomised controlled trial.

Subjects: Adults aged 18-79 years ( $n$ 1480).

Results: Individuals in cluster 1 (C1) met all recommended intakes except for red meat, those in cluster 2 (C2) met two recommendations, and those in cluster 3 (C3) and cluster 4 (C4) met one recommendation each. C1 had higher intakes of white fish, beans and lentils and low-fat dairy products and lower percentage energy intake from SFA $(P<0 \cdot 05)$. C2 consumed less chips and pizza and fried foods than C3 and C4 $(P<0 \cdot 05)$. C1 were lighter, had lower BMI and waist circumference than $\mathrm{C} 3$ and were more physically active than $\mathrm{C} 4(P<0.05)$. More individuals in $\mathrm{C} 4$ were smokers and wanted to lose weight than in $\mathrm{C} 1(P<0.05)$. Individuals who received $\mathrm{PN}$ advice in $\mathrm{C} 4$ reported greater improvements in HEI compared with $\mathrm{C} 3$ and $\mathrm{C} 1(P<0 \cdot 05)$.

Conclusions: The cluster where the fewest recommendations were met (C4) reported greater improvements in HEI following a 6-month trial of PN whereas there was no difference between clusters for those randomised to the Control, non-personalised dietary intervention.
\end{abstract}

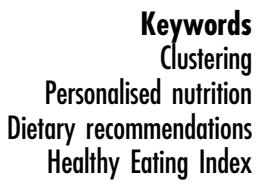

Keywords nutrition Healthy Eating Index
Global obesity prevalence has reached epidemic proportions, with $37 \%$ of men and $38 \%$ of women now either overweight or obese ${ }^{(1)}$. Poor dietary choices and inadequate physical activity are the primary causes of obesity $^{(2)}$. Current strategies for improving diet and other lifestyle behaviours, such as consuming five portions of 
fruit and vegetables per day ${ }^{(3)}$, are based on 'one size fits all' generalised dietary guidelines. Given that the burden of obesity is increasing ${ }^{(1)}$, alternative strategies for improving dietary behaviours are being developed, including predictive, personalised, preventive and participatory interventions $^{(4)}$. Recent evidence suggests that geneticbased personalised nutrition (PN) improves dietary intakes more than non-personalised advice ${ }^{(5)}$. However, since dietary intakes tend to cluster ${ }^{(6,7)}$, it may be possible to enhance the efficacy of interventions by further characterisation of participants according to their dietary and lifestyle behaviours and, subsequently, use this information to strengthen the basis for personalisation of the intervention. For example, lower intakes of fruit, vegetables and whole grains are often associated with higher intakes of red or processed meat ${ }^{(8)}$. In addition, less healthy dietary clusters are associated with increased disease risk $^{(9)}$ and unhealthy dietary and lifestyle behaviours are associated with higher levels of sedentary behaviour ${ }^{(7)}$ and mortality ${ }^{(10,11)}$. Clustering individuals based on whether they meet dietary recommendations may be a useful predictive tool for estimating response to an intervention ${ }^{(12-14)}$ and may help to stratify or personalise interventions.

The Food4Me Proof-of-Principle study was the first Internet-based study to demonstrate that PN advice was more effective in improving dietary intakes, including lowering intakes of red meat, compared with conventional 'one size fits all' population-based advice ${ }^{(15,16)}$. However, the characteristics of individuals clustered on the basis of adherence to current recommended dietary intakes of fruit and vegetables, whole grains, oily fish, dairy products and red and processed meat are unknown. Thus, the aims of the current analysis were to: (i) characterise European adults participating in the Food4Me study ${ }^{(17)}$ according to clustering based on European recommendations for healthy eating; and (ii) determine whether cluster membership predicted dietary changes following a PN intervention.

\section{Methods}

\section{Study design and population}

The Food4Me study was a 6-month, four-arm, Internetbased, randomised controlled trial (RCT) in 1607 individuals conducted across seven European countries ${ }^{(17,18)}$. Participants were recruited via the Food4Me website ${ }^{(16)}$ to emulate a web-based PN service. This was aided by local and national advertising of the study via the Internet, radio, newspapers, posters, e-flyers, social media and word of mouth. Recruitment took place between August 2012 and August 2013 in the following sites: University College Dublin (Ireland), Maastricht University (The Netherlands), University of Navarra (Spain), Harokopio University (Greece), University of Reading (UK), National Food and Nutrition Institute (Poland) and Technical University of Munich (Germany). The Research Ethics Committees at each University or Research Centre delivering the intervention granted ethical approval for the study. The Food4Me trial was registered as an RCT (NCT01530139) at Clinicaltrials. gov. All participants expressing an interest in the study were asked to sign online consent forms at two stages in the screening process.

\section{Intervention arms}

Participants were randomised to receive non-personalised, generalised dietary advice (Control) or one of three levels of PN (Level 1, Level 2 or Level 3). Briefly, non-personalised dietary advice was based on national dietary recommendations in each of the seven European countries. These 'standardised' recommendations included advice on energy intake and on the consumption of fruits and vegetables, whole grains, fish, dairy products, meat, type of fat and salt. Participants randomised to Level 1 received personalised dietary advice on how their intakes of these food groups at baseline compared with guideline amounts. Participants randomised to Level 2 received advice based on their dietary intake (as for Level 1) and also on their baseline phenotypic data. The phenotypic feedback was based on anthropometric measurements and nutrient- and metabolic-related biomarkers. Participants randomised to Level 3 received advice based on their dietary intake, phenotypic and genotypic data collected at baseline. The genotypic feedback was based on specific variants in five nutrient-responsive genes selected specifically for the study. Further details are provided elsewhere ${ }^{(17)}$.

\section{Screening questionnaires and dietary intakes}

Participants eligible for inclusion in the RCT completed an online questionnaire to collect detailed information on sociodemographic, health and anthropometric characteristics and dietary habits. Following completion of this questionnaire, participants were asked to complete an online FFQ to estimate usual dietary intake. This FFQ, which was developed and validated for the study ${ }^{(19,20)}$, included 157 food items consumed frequently in each of the seven recruitment countries. Intakes of foods and nutrients were computed in real time using a food composition database based on McCance and Widdowson's The Composition of Foods ${ }^{(21)}$. Intakes of nutrients were assessed based on standardised recommendations (see online supplementary material, Supplemental Table 1) for dietary intakes of foods and food groups ${ }^{(17)}$, which were integrated and harmonised across eight European countries (UK, Ireland, Germany, The Netherlands, Spain, Greece, Poland and Norway) ${ }^{(22-25)}$. The following four food group recommendations were used in the present analysis: (i) eat at least five portions of fruit and vegetables every day (operationalised as $\geq 400 \mathrm{~g}$ ); (ii) eat at least three portions of wholegrain products daily ( $\geq 50 \mathrm{~g}$ ); (iii) eat at least one portion of oily fish per week $(\geq 150 \mathrm{~g})$; and (iv) eat less than three portions of red or processed meat 
per week $(\leq 450 \mathrm{~g})^{(17)}$. The Healthy Eating Index 2010 (HEI) was derived based on intakes of the following components: ratio of MUFA + PUFA to SFA, protein, salt, 'empty calories', refined grains, seafood and plant protein, fruit, whole fruit, vegetables, greens and beans, whole grains and dairy products ${ }^{(26)}$.

\section{Personalised feedback report}

Participants randomised to $\mathrm{PN}$ received personalised reports via email at baseline, month 3 and month 6 of the intervention based on diet, anthropometric measurements and physical activity. Using information on the individual's intakes of nutrients, algorithms were used to rank information on need for dietary change and to provide participants with three specific dietary, food-based goals. For participants randomised to Level 2 and Level 3, the dietary advice was also based on phenotypic data (Level 2) and phenotypic plus genotypic data (Level 3). Reported intakes were compared with recommended intakes and determined to be adequate, high or low. If intakes were too high or too low, contributing foods were identified and specific messages developed to advise change in intake of those foods. Dietary intakes relative to recommendations were illustrated using a threecolour sliding scale: green representing 'Good, no change recommended', amber representing 'Improvement recommended' and red representing 'Improvement strongly recommended'. For the genotype-based information, risk was indicated using 'Yes' or 'No' according to whether the participant did or did not carry the higher-risk variant for each of the five nutrient-related genes included in the study. Additionally, each report contained a personalised message from the dietitian/nutritionist to the participant. Further details of the protocol are provided elsewhere ${ }^{(17)}$.

\section{Antbropometric, sociodemographic and physical activity measures}

Detailed standardised online instructions were given for participants to self-measure and self-report their body weight, height and waist circumference via the Food4Me website $^{(16)}$. BMI was estimated from body weight and height. Self-reported measurements were validated in a sub-sample of the participants ( $n$ 140) and showed a high degree of reliability ${ }^{(27)}$. Physical activity level and time spent in sedentary behaviours were estimated from triaxial accelerometers (TracmorD, Philips Consumer Lifestyle, The Netherlands). Participants self-reported smoking habits and occupation. Occupations were grouped according to the European classifications of occupations and their salaries (the European-wide average salary for each occupation was compared with the mean overall salary; if the SD of the salary was $>0.5$, they were placed in Group 1, between 0.5 to -0.5 were placed into Group 2, and $<-0.5$ were placed into Group 3) as follows: Group 1, Professional and managerial (professionals; managers); Group 2, Intermediate (armed forces occupations; technicians and associate professionals; clerical support workers); Group 3, Routine and manual (craft and related trades workers; plant and machine operators and assemblers; service and sales workers; elementary occupations; skilled agricultural, forestry and fishery workers) ${ }^{(28,29)}$. Categories for 'Students' and 'Retired and unemployed' were added.

\section{Statistical analysis}

Data were analysed using the statistical software packages Stata version 13 and IBM SPSS Statistics Version 22.0. Clusters of dietary recommendations were generated based on whether participants met the following four food group recommendations at baseline and were coded as 0 or 1 accordingly: (i) eat at least five portions of fruit and vegetables every day (operationalised as $\geq 400 \mathrm{~g}$ ); (ii) eat at least three portions of wholegrain products daily ( $\geq 50 \mathrm{~g}$ ); (iii) eat at least one portion of oily fish per week $(\geq 150 \mathrm{~g})$; and (iv) eat less than three portions of red or processed meat per week $(\leq 450 \mathrm{~g})$. Clusters were derived using the SPSS Two Step cluster analysis procedure ${ }^{(30)}$. Small pre-clusters were generated based on the log-likelihood distance criterion (Step 1) and were merged into distinct groups using agglomerative hierarchical clustering (Step 2). Automatic selection and the Bayesian information criterion were used to determine the optimal number of clusters. Robustness and stability of the final clusters were re-evaluated by random ordering of cases (four times). This clustering methodology identified the percentage of participants within each cluster who met recommended intakes of each of the four food groups of public health importance. Logistic regression was used to test for significant differences across categorical variables and ANOVA was used for continuous variables. Tukey pairwise comparisons were used to test for significant differences between clusters. Analyses were adjusted for age, sex, country, BMI, physical activity level and smoking, except when those (or related) variables were being assessed (i.e. analyses were not adjusted for BMI when assessing BMI, body weight or waist circumference). Results were deemed significant at $P<0.05$. To exclude extreme intakes of the food groups used for clustering, the top and bottom 3 SD of these intakes were excluded prior to clustering.

\section{Results}

Of the 5562 individuals who registered on the Food4Me website, 1607 were randomised into the study and a total of 1480 provided baseline data on dietary intakes ${ }^{(17)}$.

\section{Dietary adequacies across Food4Me cobort}

Recommended intakes for nutrients are summarised in the online supplementary material, Supplemental Table 1 . On average, $50 \%$ of individuals met the recommendations for 
total fat (Supplemental Table 2). The percentage of individuals who met the recommendations for SFA, MUFA and PUFA intake was 54, 24 and 36\%, respectively. Only $56 \%$ of individuals met the recommendation for carbohydrate intake, whereas $91 \%$ of individuals had adequate protein intakes. Only 7 and $46 \%$ of individuals met the recommendations for salt and dietary fibre intakes, respectively. Meeting recommended micronutrient intakes ranged from $61 \%$ (folate) to $99 \%$ (vitamin $\mathrm{B}_{12}$ ).

As summarised in the online supplementary material, Supplemental Table 2, approximately half (52\%) of participants reported consuming at least five portions of fruit and vegetables per day and $32 \%$ consumed at least one portion of oily fish per week. Nearly three-quarters (74\%) of participants consumed more than three servings of whole grains per day and approximately half of participants (51\%) consumed less than three servings of red meat per week. Fourteen per cent of individuals met the recommendation for dairy product intake $(\geq 600 \mathrm{~g} / \mathrm{d})$.

\section{Cluster characterisation}

Clustering of individuals according to whether they met the intake recommendations for dairy products, fruits and vegetables, oily fish, red meat and whole grains at baseline did not create clear clustering due to the low percentage of individuals who met the recommendation for dairy products (two clusters). Exclusion of dairy products as a clustering variable provided improved clustering, as estimated by the silhouette measure of cohesion and separation (average silhouette: $0.3 v$. 0.5; four clusters; see online supplementary material, Supplemental Table 3). Cluster 1 (C1) was the largest ( $n$ 475) and was particularly characterised by individuals meeting the recommended intake for oily fish (100\% of individuals); 74 and $69 \%$ of $\mathrm{C} 1$ members met the recommendations for whole grains and fruit and vegetables, respectively, whereas only $46 \%$ met the recommendation for red meat. Cluster 2 (C2; $n$ 398) was the second largest and was particularly characterised by all members meeting recommendations for whole grains (100\%) and red meat (100\%), only $50 \%$ meeting the recommendation for fruit and vegetables and no one meeting the recommendation for oily fish. All individuals in cluster 3 (C3; $n$ 348) met the recommendation for whole grains, but no one met the recommendation for oily fish or red meat, whereas only $48 \%$ met the recommended intake for fruit and vegetables. None of the participants in cluster 4 (C4; $n$ 259) met the recommended intake for either oily fish or whole grains; only 50 and $29 \%$ of C4 members achieved the recommended intakes for red meat and fruit and vegetables, respectively (Supplemental Table 3).

\section{Dietary intakes by cluster}

Intakes of oily fish and fruit and vegetables were higher in $\mathrm{C} 1$ than in $\mathrm{C} 2, \mathrm{C} 3$ and $\mathrm{C} 4(P<0 \cdot 05)$, and wholegrain intakes were higher in C1, C2 and C3 than in C4 (Table 1; $P<0.05)$. Red meat intake was lower in C1, C2 and C3 than in $\mathrm{C} 4(P<0.05)$. Intakes of fruit juice, eggs, chicken, white fish, fish products, beans and lentils and low-fat dairy products were higher in $\mathrm{C} 1$ than $\mathrm{C} 4$, whereas intakes of non-wholegrain products were lower $(P<0 \cdot 05)$. Participants in $\mathrm{C} 2$ had lower intakes of chips and pizza and fried foods than C3 and C4 $(P<0.05$; Table 1$)$. Total energy intake and ratio of energy intake to BMR were higher in $\mathrm{C} 1$ than in $\mathrm{C} 2$ and $\mathrm{C} 4$ and higher in C3 than in C2 $(P<0 \cdot 05$; Table 1$)$. Individuals in $\mathrm{C} 1$ derived higher percentages of energy intake from PUFA and protein than those in $\mathrm{C} 2$ and $\mathrm{C} 4(P<0.05)$ and individuals in $\mathrm{C} 2$ derived a higher percentage energy from carbohydrates than participants in $\mathrm{C} 3$ and $\mathrm{C} 4(P<0.05)$. In contrast, individuals in $\mathrm{C} 1$ had lower percentage energy intakes from total fat and SFA than those in $\mathrm{C} 4(P<0.05)$ and higher percentage energy intake from MUFA than participants in $\mathrm{C} 2$ and $\mathrm{C} 3(P<0.05)$. Individuals in $\mathrm{C} 1$ had lower percentage energy intake from sugar than those in $\mathrm{C} 2$ $(P<0.05)$. Participants in $\mathrm{C} 1$ consumed more dietary fibre and salt than those in $\mathrm{C} 2$ and $\mathrm{C} 4(P<0 \cdot 05)$.

More individuals in $\mathrm{C} 1$ met the recommendations for total fat intake (51\%), SFA (62\%), PUFA (42\%) and dietary fibre (56\%) than C4 cluster members (see online supplementary material, Supplemental Table 4). Fewer individuals in $\mathrm{C} 1$ met the recommendation for protein intake (86\%) than those in C2 (97\%) and C3 (93\%). Furthermore, fewer individuals in $\mathrm{C} 1$ met the recommendation for salt intake (5\%) than C2 (11\%) and C4 (17\%; Supplemental Table 4).

\section{Sociodemographic, anthropometric and bealth characteristics by cluster}

Individuals in $\mathrm{C} 1$ were on average 4.5 years older than those in $\mathrm{C} 4 \quad(P<0.05$; Table 2$)$. Body weight was significantly lower in $\mathrm{C} 1$ than in $\mathrm{C} 3$, and in C2 compared with C3 and C4 $(P<0.05)$. Individuals in C1 had $1.4 \mathrm{~kg} / \mathrm{m}^{2}$ lower BMI and $5.0 \mathrm{~cm}$ lower waist circumference than participants in C3 $(P<0.05)$ and physical activity level was higher in C1 than $\mathrm{C} 2$ and $\mathrm{C} 4(P<0 \cdot 05)$. Eleven per cent more individuals in $\mathrm{C} 4$ wanted to lose weight than in $\mathrm{C} 1(P<0 \cdot 05$; Table 2$)$ and $\mathrm{C} 4$ was characterised by more current smokers than $\mathrm{C} 1$ $(P<0.05)$. Twelve per cent more individuals in $\mathrm{C} 1$ than in $\mathrm{C} 4$ had a professional or managerial occupation, and similarly $7 \%$ more individuals had a manual occupation in C4 compared with C1 $(P<0.05$; Table 2$)$. No other significant differences were observed (Table 2 ).

\section{Changes in Healthy Eating Index by cluster after 6-month intervention}

Baseline and follow-up HEI scores and their components are presented in Table 3. There were no significant differences in changes in HEI between clusters for those randomised to non-personalised dietary advice. In contrast, for individuals who received PN advice (based 
Table 1 Food and nutrient and intakes by cluster of adherence to dietary recommendations at baseline among adults aged 18-79 years ( $n$ 1480), Food4Me study

\begin{tabular}{|c|c|c|c|c|c|c|c|c|c|}
\hline & \multicolumn{8}{|c|}{ Cluster } & \multirow[b]{3}{*}{$P^{\star}$} \\
\hline & \multicolumn{2}{|c|}{ C1 ( $n$ 475) } & \multicolumn{2}{|c|}{ C2 ( $n$ 398) } & \multicolumn{2}{|c|}{ C3 ( $n$ 348) } & \multicolumn{2}{|c|}{ C4 (n 259) } & \\
\hline & Mean & SD & Mean & SD & Mean & SD & Mean & SD & \\
\hline \multicolumn{10}{|l|}{ Dietary recommendations $(\mathrm{g} / \mathrm{d})$} \\
\hline Oily fish & $48^{2,3,4}$ & 32 & 8 & 7 & 10 & 7 & 8 & 7 & $<0.001$ \\
\hline Whole grains & $183^{2,4}$ & 182 & $216^{3,4}$ & 184 & $205^{4}$ & 165 & 22 & 16 & $<0.001$ \\
\hline Red meat & $85^{2,3,4}$ & 81 & $30^{3,4}$ & 20 & $119^{4}$ & 53 & 84 & 96 & $<0.001$ \\
\hline Fruit and vegetables & $610^{2,3,4}$ & 371 & $470^{3,4}$ & 303 & 456 & 288 & 339 & 218 & $<0.001$ \\
\hline \multicolumn{10}{|l|}{ Other food intakes $(\mathrm{g} / \mathrm{d})$} \\
\hline Fruit juice & $117^{3,4}$ & 181 & 114 & 165 & 94 & 144 & 76 & 108 & 0.008 \\
\hline Non-wholemeal & $116^{2,4}$ & 140 & $78^{4}$ & 76 & $114^{4}$ & 103 & 149 & 189 & $<0.001$ \\
\hline Eggs & $41^{2,3}$ & 41 & 22 & 24 & 31 & 47 & 30 & 51 & $<0.001$ \\
\hline Chicken, grilled or roast & $36^{2,3,4}$ & 37 & $17^{3}$ & 21 & 28 & 25 & 25 & 27 & $<0.001$ \\
\hline White fish & $26^{2,3,4}$ & 26 & 10 & 14 & 13 & 14 & 11 & 14 & $<0.001$ \\
\hline Fish products & $19^{2,4}$ & 30 & $10^{3}$ & 11 & 14 & 16 & 13 & 15 & $<0.001$ \\
\hline Beans and lentils & $30^{2,3}$ & 40 & 15 & 24 & 16 & 27 & 22 & 28 & $<0.001$ \\
\hline Butter & $4^{3}$ & 9 & $6^{3}$ & 11 & $9^{4}$ & 18 & 5 & 12 & 0.005 \\
\hline Low-fat dairy & $293^{2,3,4}$ & 296 & 217 & 203 & 221 & 212 & 173 & 219 & $<0.001$ \\
\hline High-fat dairy & 64 & 120 & 60 & 119 & 83 & 113 & 83 & 204 & 0.44 \\
\hline Sugar-sweetened beverages & 36 & 176 & 18 & 55 & 40 & 139 & 41 & 84 & 0.39 \\
\hline Low-calorie soft drinks & 66 & 194 & 46 & 154 & 80 & 239 & 72 & 190 & 0.53 \\
\hline Added sugar & 4 & 9 & 4 & 11 & 5 & 13 & 7 & 13 & 0.11 \\
\hline Chocolate and sweets & 21 & 37 & 19 & 23 & 26 & 61 & 17 & 26 & 0.10 \\
\hline Cakes & 22 & 31 & 18 & 25 & 20 & 25 & 22 & 39 & 0.08 \\
\hline Biscuits & 30 & 55 & 21 & 37 & 35 & 88 & 27 & 55 & 0.38 \\
\hline Ice cream & 7 & 19 & 6 & 11 & 7 & 12 & 7 & 13 & 0.62 \\
\hline Pastries & 8 & 34 & 4 & 6 & 6 & 10 & 10 & 39 & 0.49 \\
\hline Crisps & 4 & 10 & $3^{3}$ & 5 & 5 & 10 & 4 & 8 & 0.06 \\
\hline Chips and pizza & 30 & 41 & $24^{3,4}$ & 22 & 35 & 30 & 34 & 35 & 0.001 \\
\hline Fried foods & $33^{2}$ & 52 & $21^{3,4}$ & 28 & 34 & 35 & 33 & 30 & 0.047 \\
\hline \multicolumn{10}{|l|}{ Nutrient intakes } \\
\hline Total energy $(\mathrm{MJ} / \mathrm{d})$ & $12 \cdot 02^{2,4}$ & $5 \cdot 10$ & $9 \cdot 29^{3}$ & $3 \cdot 12$ & $11.95^{4}$ & 4.46 & 8.82 & $4 \cdot 10$ & $<0.001$ \\
\hline Total energy $(\mathrm{kcal} / \mathrm{d})$ & $2870^{2,4}$ & 1219 & $2218^{3}$ & 745 & $2855^{4}$ & 1065 & 2106 & 978 & $<0.001$ \\
\hline El:BMR & $1 \cdot 9^{2,4}$ & 0.7 & $1.5^{3}$ & 0.5 & $1 \cdot 8^{4}$ & 0.6 & 1.4 & 0.6 & $<0.001$ \\
\hline Total fat (\% energy) & $36 \cdot 0^{2,4}$ & $5 \cdot 7$ & $34 \cdot 1^{3,4}$ & $5 \cdot 6$ & $36 \cdot 4$ & 5.5 & 37.9 & $6 \cdot 6$ & $<0.001$ \\
\hline SFA (\% energy) & $13 \cdot 4^{3,4}$ & $2 \cdot 8$ & $13 \cdot 6^{3,4}$ & 3.3 & 14.9 & 3.0 & $15 \cdot 3$ & 3.3 & $<0.001$ \\
\hline MUFA (\% energy) & $14 \cdot 2^{2,3}$ & $3 \cdot 2$ & $12 \cdot 6^{3,4}$ & $2 \cdot 8$ & $13 \cdot 6^{4}$ & 2.6 & $14 \cdot 8$ & 3.5 & $<0.001$ \\
\hline PUFA (\% energy) & $6 \cdot 0^{2,4}$ & 1.4 & 5.7 & 1.4 & 5.6 & 1.3 & 5.5 & 1.7 & 0.003 \\
\hline Protein (\% energy) & $18 \cdot 3^{2,3,4}$ & $4 \cdot 1$ & $15 \cdot 5^{3,4}$ & $3 \cdot 2$ & $17 \cdot 0$ & 2.9 & $17 \cdot 3$ & 3.7 & $<0.001$ \\
\hline Carbohydrate (\% energy) & $44 \cdot 5^{2,3}$ & 7.5 & $49 \cdot 6^{3,4}$ & $7 \cdot 0$ & $45 \cdot 6$ & $6 \cdot 4$ & 43.7 & $8 \cdot 3$ & $<0.001$ \\
\hline Sugars (\% energy) & $21 \cdot 0^{2}$ & 5.9 & $22 \cdot 5^{3,4}$ & $6 \cdot 1$ & $19 \cdot 8$ & $5 \cdot 6$ & $20 \cdot 8$ & 5.9 & $<0.001$ \\
\hline Dietary fibre $(\mathrm{g} / \mathrm{d}) \dagger$ & $34 \cdot 0^{2,4}$ & $15 \cdot 8$ & $30 \cdot 2^{4}$ & 14.4 & $31 \cdot 7^{4}$ & $12 \cdot 8$ & 18.7 & 8.2 & $<0.001$ \\
\hline Salt $(q / d) t$ & $8 \cdot 3^{2,4}$ & 4.0 & $6 \cdot 1^{3}$ & 2.7 & $8 \cdot 7^{4}$ & 3.6 & 5.9 & 3.6 & $<0.001$ \\
\hline
\end{tabular}

El:BMR, ratio of energy intake to BMR.

${ }^{\star}$ ANOVA was used to test for significant differences across clusters. Models were adjusted for age, sex, BMI, physical activity level, smoking habits and country; significant $P$ values are highlighted in bold. Post hoc Tukey tests were performed to test for significant differences between clusters; superscript numbers denote where the differences lie across the clusters. For example, 2 indicates that the mean value is significantly different from that in cluster $2(P<0.05)$.

$\dagger P$ values are also adjusted for total energy intake.

on information of current diet alone or combined with information on phenotype and genotype), changes in HEI differed between clusters $(P<0 \cdot 001)$. There were bigger improvements in HEI for participants in C4 compared with $\mathrm{C} 1$ and $\mathrm{C} 2 \quad(P<0.05)$ and in $\mathrm{C} 2$ compared with $\mathrm{C} 4$ $(P<0.05$; Fig. 1$)$. There were no significant differences in changes in HEI between clusters when PN was stratified by Level 1, Level 2 or Level 3 (data not shown).

\section{Sensitivity analyses}

Exclusion of participants with reported intakes more than 3 SD above or below the mean dietary intakes of whole grains, oily fish, red meat and fruit and vegetables revealed similar clusters (see online supplementary material, Supplemental Table 5). The pattern of the main results remained the same, with individuals in C3 and C4 making greater changes in HEI at month 6 than those in $\mathrm{C} 1$, as did participants in $\mathrm{C} 4$ compared with those in $\mathrm{C} 2(P<0.05)$.

\section{Discussion}

\section{Main findings}

Based on our secondary analysis in the Food4Me Proof-ofPrinciple study, we identified four distinct clusters of 
Table 2 Sociodemographic characteristics by cluster of adherence to dietary recommendations at baseline among adults aged 18-79 years (n 1480), Food4Me study

\begin{tabular}{|c|c|c|c|c|c|c|c|c|c|}
\hline & \multicolumn{8}{|c|}{ Cluster } & \multirow[b]{3}{*}{$P^{\star}$} \\
\hline & \multicolumn{2}{|c|}{ C1 (n 475) } & \multicolumn{2}{|c|}{ C2 (n 398) } & \multicolumn{2}{|c|}{ C3 (n 348) } & \multicolumn{2}{|c|}{ C4 (n 259) } & \\
\hline & Mean & SD & Mean & SD & Mean & SD & Mean & SD & \\
\hline Age (years) & $41 \cdot 2^{2,3}$ & $12 \cdot 7$ & $39 \cdot 2^{4}$ & $14 \cdot 2$ & $41 \cdot 2^{4}$ & $12 \cdot 7$ & $36 \cdot 7$ & 11.5 & $<0.001$ \\
\hline $\begin{array}{l}\text { Female (\%) } \\
\text { Ethnicity }(\%)\end{array}$ & \multicolumn{2}{|c|}{56.0} & \multicolumn{2}{|c|}{67.3} & \multicolumn{2}{|c|}{$47 \cdot 4$} & \multicolumn{2}{|c|}{64.1} & 0.79 \\
\hline Caucasian & \multicolumn{2}{|c|}{$95 \cdot 6$} & \multicolumn{2}{|c|}{$96 \cdot 5$} & \multicolumn{2}{|c|}{$97 \cdot 7$} & \multicolumn{2}{|c|}{$98 \cdot 1$} & 0.16 \\
\hline Occupation (\%) & \multirow{2}{*}{\multicolumn{2}{|c|}{$44.2^{4}$}} & \multirow{2}{*}{\multicolumn{2}{|c|}{37.8}} & \multirow{2}{*}{\multicolumn{2}{|c|}{39.4}} & \multirow{2}{*}{\multicolumn{2}{|c|}{$32 \cdot 2$}} & \\
\hline Professional and managerial & & & & & & & & & 0.014 \\
\hline Intermediate occupations & \multicolumn{2}{|c|}{$25 \cdot 9$} & & & \multicolumn{2}{|c|}{28.5} & \multicolumn{2}{|c|}{28.7} & 0.16 \\
\hline Routine and manual & \multicolumn{2}{|c|}{$7 \cdot 4^{4}$} & & & \multicolumn{2}{|c|}{$12 \cdot 9$} & \multicolumn{2}{|c|}{$14 \cdot 3$} & 0.006 \\
\hline Student & \multicolumn{2}{|c|}{$13 \cdot 5$} & & & \multicolumn{2}{|c|}{9.5} & \multicolumn{2}{|c|}{$14 \cdot 7$} & 0.18 \\
\hline Not currently working & \multirow{2}{*}{\multicolumn{2}{|c|}{$9 \cdot 1$}} & & & \multicolumn{2}{|c|}{$9 \cdot 8$} & & & 0.38 \\
\hline Anthropometrics & & & & & & & & & \\
\hline Body weight $(\mathrm{kg})$ & $74 \cdot 6^{3}$ & $15 \cdot 1$ & $70 \cdot 5^{3,4}$ & $15 \cdot 0$ & $80 \cdot 3^{4}$ & $16 \cdot 0$ & $74 \cdot 1$ & $16 \cdot 3$ & $<0.001$ \\
\hline $\mathrm{BMI}\left(\mathrm{kg} / \mathrm{m}^{2}\right)$ & $25 \cdot 4^{2,3}$ & 4.4 & $24 \cdot 1^{3,4}$ & 4.4 & $26 \cdot 8$ & 4.9 & $26 \cdot 0$ & 5.7 & $<0.001$ \\
\hline Waist circumference $(\mathrm{cm})$ & $85 \cdot 4^{3}$ & $13 \cdot 0$ & 81.8 & 13.2 & 90.4 & $14 \cdot 1$ & $85 \cdot 9$ & $14 \cdot 1$ & $<0.001$ \\
\hline Physical activity & & & & & & & & & \\
\hline Physical activity level & $1 \cdot 8^{2,4}$ & 0.2 & $1 \cdot 7^{3}$ & 0.2 & $1 \cdot 8^{4}$ & 0.2 & $1 \cdot 7$ & 0.2 & $<0.001$ \\
\hline Sedentary behaviour ( $\min / \mathrm{d})$ & 746 & 73 & 742 & 77 & 750 & 76 & 744 & 7 & 0.96 \\
\hline Dietary conditions (\%) & & & & & & & & & \\
\hline Want to lose weight & & & & & & & & & 0.013 \\
\hline Restricted diet & & & & & & & & & 0.47 \\
\hline Medication use (\%) & & & & & & & & & \\
\hline Prescribed medication & & & & & & & & & 0.79 \\
\hline Non-prescribed medication & & & & & & & & & 0.18 \\
\hline Health and disease & & & & & & & & & \\
\hline Current smoker (\%) & & & & & & & & & 0.005 \\
\hline Total cholesterol (mmol/l) & $4 \cdot 6$ & 0.9 & 4.5 & 1.0 & 4.7 & 1.0 & 4.6 & 0.9 & 0.09 \\
\hline High blood pressure (\%) & & & & & & & & & 0.89 \\
\hline Heart disease (\%) & & & & & & & & & 0.17 \\
\hline
\end{tabular}

Values are presented means and standard deviations or percentages.

*ANOVA and logistic regression were used to test for significant differences across clusters in continuous and categorical variables, respectively. Analyses were adjusted for age, sex, BMI, physical activity level, smoking habits and country; significant $P$ values are highlighted in bold. Post hoc Tukey tests (continuous data) and logistic regression (categorical) were used to test for significant differences between clusters; superscript numbers denote where the differences lie across the clusters. For example, 2 indicates that the mean value or percentage is significantly different from that in cluster $2(P<0.05)$.

individuals according to their adherence to current European dietary recommendations. Individuals in $\mathrm{C} 1$ and C2 met more dietary recommendations than those in C3 and C4. Moreover, on average, individuals in C1 and C2 had a healthier diet, lower BMI and waist circumference, and smoked less, compared with those in C3 and C4. When randomised to a 6-month $\mathrm{PN}$ intervention, participants in C4 made the greatest improvements in their diets (as estimated by HEI), compared with participants receiving non-personalised 'one size fits all' generalised advice. The present study is the first to investigate clusters of adherence to European dietary recommendations and to determine the responsiveness of cluster members to $\mathrm{PN}$ advice.

\section{Comparison with other studies}

Previous studies have used cluster analysis to categorise individuals $^{(31)}$. We used cluster analysis to categorise individuals based on their adherence to current European food-based dietary guidelines at baseline for participants in the Food4Me intervention study. This approach identified groups of individuals who differed in the number, and groupings, of dietary recommendations they met. Clusters where more individuals met the recommendations were characterised by being slightly older and in more highly educated occupations, which is a well-established characteristic of healthy dietary clusters ${ }^{(32)}$.

Clustering of dietary intakes and adequacies has been investigated in relation to several health outcomes ${ }^{(7,8,33)}$ and can be strong predictors of these outcomes ${ }^{(34)}$. A recent review of dietary clusters and health outcomes by the US Department of Agriculture ${ }^{(35)}$ concluded that the strongest evidence for an association between unhealthy dietary patterns and increased disease risk is for CVD, followed by obesity and then type 2 diabetes. The review concluded that there was a lack of studies assessing dietary intakes at follow-up and using a universal and quantitative indicator of dietary intake. Our study is in line with these recommendations as we utilised the HEI, which is a validated estimate of dietary adequacy, and we assessed dietary change using the same instrument at both baseline and follow-up. Although more limited, some prospective and RCT studies have investigated the effect of clustering on changes in health outcomes ${ }^{(12,36,37)}$ and 
Table 3 Healthy Eating Index 2010 (HEI) score and its constituents at baseline and month 6 by cluster of adherence to dietary recommendations among adults aged $18-79$ years $(n$ 1480), Food4Me study

\begin{tabular}{|c|c|c|c|c|c|c|c|c|c|}
\hline & \multicolumn{8}{|c|}{ Cluster } & \multirow[b]{3}{*}{$P^{\star}$} \\
\hline & \multicolumn{2}{|c|}{ C1 (n 475) } & \multicolumn{2}{|c|}{ C2 (n 398) } & \multicolumn{2}{|c|}{ C3 (n 348) } & \multicolumn{2}{|c|}{ C4 (n 259) } & \\
\hline & Mean & SD & Mean & SD & Mean & SD & Mean & SD & \\
\hline \multicolumn{10}{|l|}{ Baseline score } \\
\hline Total HEI & $53 \cdot 3^{2,3,4}$ & 8.9 & $50 \cdot 5^{3,4}$ & 8.9 & $47 \cdot 5^{4}$ & 8.9 & 41.8 & $10 \cdot 1$ & $<0.001$ \\
\hline Fatty acid ratio† & $3 \cdot 2^{2,3,4}$ & $2 \cdot 4$ & $2 \cdot 2^{3,4}$ & 2.4 & 1.7 & $1 \cdot 7$ & 2.0 & $2 \cdot 0$ & $<0.001$ \\
\hline Protein & $3 \cdot 7^{2,3,4}$ & 0.7 & $3 \cdot 2^{3,4}$ & 0.6 & 3.5 & 0.6 & 3.5 & 0.7 & $<0.001$ \\
\hline Salt & 0.1 & 0.5 & 0.1 & 0.7 & 0.1 & 0.6 & 0.1 & 0.6 & 0.002 \\
\hline Empty calories & $8 \cdot 8^{2}$ & 4.0 & $7 \cdot 7$ & $4 \cdot 3$ & 8.5 & 3.8 & 7.5 & $4 \cdot 1$ & 0.012 \\
\hline Refined grains & $6 \cdot 1^{2,3,4}$ & 3.7 & $4 \cdot 8$ & 3.7 & 4.4 & 3.7 & 4.7 & 4.0 & $<0.001$ \\
\hline Seafood and plant protein & $5 \cdot 0^{2,3,4}$ & 0.2 & 4.5 & 1.0 & $4 \cdot 3$ & 1.1 & 4.4 & 1.1 & $<0.001$ \\
\hline Fruit & $3.8^{3}$ & 1.3 & $3 \cdot 8^{3}$ & 1.4 & $3 \cdot 3$ & 1.5 & 3.3 & 1.5 & $<0.001$ \\
\hline Whole fruit & $4 \cdot 2^{3,4}$ & 1.3 & $4 \cdot 1^{3,4}$ & 1.3 & 3.6 & 1.5 & 3.6 & 1.6 & $<0.001$ \\
\hline Vegetables & $2 \cdot 5^{3,4}$ & $1 \cdot 1$ & $2 \cdot 3^{3,4}$ & $1 \cdot 1$ & $2 \cdot 0$ & 0.9 & $2 \cdot 1$ & $1 \cdot 1$ & $<0.001$ \\
\hline Greens and beans & $4 \cdot 2^{2,3,4}$ & $1 \cdot 1$ & $3.8^{3}$ & 1.3 & 3.5 & 1.3 & 3.7 & 1.4 & $<0.001$ \\
\hline Whole grains & $7 \cdot 3$ & 3.5 & 9.5 & $1 \cdot 2$ & $8 \cdot 8$ & 1.9 & $2 \cdot 9$ & $2 \cdot 2$ & $<0.001$ \\
\hline Dairy products & $4 \cdot 7^{2,3,4}$ & 2.6 & $4 \cdot 7^{4}$ & $2 \cdot 7$ & $4 \cdot 3^{4}$ & $2 \cdot 2$ & 4.4 & $2 \cdot 7$ & 0.27 \\
\hline \multicolumn{10}{|l|}{ Follow-up score } \\
\hline Total HEI & $55 \cdot 7^{2,3,4}$ & $9 \cdot 1$ & $53 \cdot 3^{4}$ & 9.6 & 51.4 & 8.7 & 48.0 & $10 \cdot 3$ & $<0.001$ \\
\hline Fatty acid ratio† & $3 \cdot 8^{2,3,4}$ & 2.6 & $3 \cdot 1^{3}$ & $2 \cdot 7$ & 2.5 & $2 \cdot 1$ & 2.6 & $2 \cdot 2$ & $<0.001$ \\
\hline Protein & $3 \cdot 8^{2,3,4}$ & 0.7 & $3 \cdot 3^{3,4}$ & 0.6 & 3.6 & 0.6 & 3.6 & 0.6 & $<0.001$ \\
\hline Salt & 0.1 & 0.6 & $0 \cdot 2^{3}$ & 0.9 & $0 \cdot 1$ & 0.6 & 0.1 & 0.6 & 0.002 \\
\hline Empty calories & $8 \cdot 7^{2}$ & 4.0 & 7.4 & $4 \cdot 1$ & $8 \cdot 8$ & 4.0 & 8.1 & $4 \cdot 1$ & 0.002 \\
\hline Refined grains & $6 \cdot 2^{4}$ & 3.8 & 5.4 & 3.8 & $5 \cdot 1$ & 3.8 & 4.9 & 3.8 & 0.004 \\
\hline Seafood and plant protein & $5 \cdot 0^{2,3}$ & 0.2 & 4.7 & 0.8 & 4.6 & 1.0 & 4.7 & 0.9 & $<0.001$ \\
\hline Fruit & $4 \cdot 1$ & 1.3 & $4 \cdot 2^{3}$ & 1.2 & 3.7 & 1.4 & 3.7 & 1.5 & 0.009 \\
\hline Whole fruit & 4.4 & 1.2 & 4.4 & $1 \cdot 1$ & $4 \cdot 1$ & 1.4 & 4.0 & 1.5 & 0.023 \\
\hline Vegetables & $2 \cdot 8^{3,4}$ & 1.2 & $2 \cdot 7^{3,4}$ & 1.3 & $2 \cdot 3$ & 1.0 & 2.4 & 1.0 & $<0.001$ \\
\hline Greens and beans & $4 \cdot 3^{2,3}$ & 1.0 & $4 \cdot 0$ & 1.2 & 3.9 & 1.2 & 4.1 & 1.2 & 0.001 \\
\hline Whole grains & $7 \cdot 9^{2,3,4}$ & 3.1 & $9 \cdot 2^{4}$ & 1.9 & $8 \cdot 5^{4}$ & $2 \cdot 7$ & 5.5 & 3.7 & $<0.001$ \\
\hline Dairy products & 4.8 & $2 \cdot 7$ & 4.7 & 2.8 & 4.4 & $2 \cdot 3$ & 4.5 & $2 \cdot 6$ & 0.52 \\
\hline
\end{tabular}

*Fatty acid ratio is the ratio of unsaturated fatty acids (mono- and polyunsaturated fatty acids) to saturated fatty acids.

*ANOVA was used to test for significant differences across clusters. Models were adjusted for age, sex, BMI, physical activity level, smoking habits and country; significant $P$ values are highlighted in bold. Post hoc Tukey tests were used to test for significant differences between clusters; superscript numbers denote where the differences lie across the clusters. For example, 2 indicates that the mean value is significantly different from that in cluster 2 $(P<0.05)$.

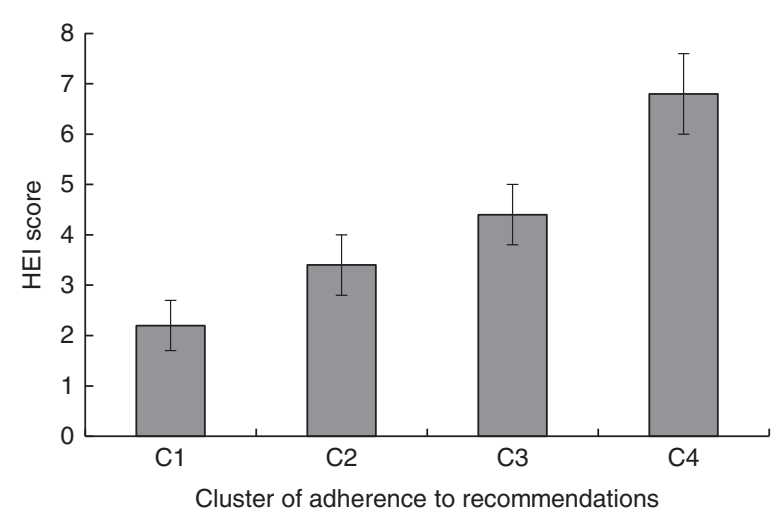

Fig. 1 Changes from baseline to month 6 in Healthy Eating Index 2010 (HEI) score by cluster of adherence to dietary recommendations at baseline among adults aged $18-79$ years $(n 1480)$, Food4Me study. Values are predicted means with their standard errors represented by vertical bars. Models were adjusted for age, sex, BMI, physical activity level, smoking habits and country $\left(P_{\text {trend }}<0.001\right)$; post hoc Tukey tests were used to test for significant differences between clusters (C); C4>C1 $(P<0.001)$, C3 $>\mathrm{C} 1$ $(P=0.005)$ some studies have used adherence to dietary recommendations to derive clusters ${ }^{(12-14,38,39)}$. Dietary recommendations used in studies included in the systematic review by the US Department of Agriculture ${ }^{(35)}$ varied according to the study, but all included a measure of fruit and vegetable, wholegrain and meat intake.

To our knowledge, no previous research has evaluated the impact of clustering of dietary recommendations on the response to a PN intervention. We observed that individuals in the cluster where the fewest recommendations were met $(\mathrm{C} 4)$ reported the biggest improvement in HEI following PN intervention but there were no differences between clusters in response to conventional, non-personalised dietary advice. Given that adverse lifestyle behaviours and the prevalence and risk of death from obesity-related diseases are strongly socio-economically patterned $^{(40)}$, it is important that appropriate interventions are targeted to those most in need of improved lifestyle. While research on the development and implementation of PN interventions and their effects on changing diets is in its infancy ${ }^{(41)}$, the findings from the present study provide 
encouragement that PN interventions can be more effective than current 'one size fits all' interventions and that they may be particularly effective among individuals with the poorest diets. There have been concerns that PN may be taken up only by the 'worried well'(42), who already have adequate dietary intakes. However, our findings suggest that PN is most effective in people who have the least adequate diets, and therefore the greatest need for improvement in dietary intakes, with the potential for significant reductions in disease risk.

\section{Strengths and limitations}

The present study had a number of strengths. Our findings are derived from a relatively large number of participants who were broadly representative of European adults from seven different European countries ${ }^{(18)}$. The Food4Me RCT collected extensive information on anthropometrics, physical activity and sociodemographic and health-related data, which contributed to detailed characterisation of participants in the clusters. Our study design allowed us to estimate changes in dietary intakes using the same validated instrument at baseline and at month 6. Furthermore, we quantified responses using the HEI, which has been shown to be an effective indicator of overall diet quality ${ }^{(26)}$ and, therefore, a better measure of overall dietary change than outcomes based on single foods or nutrients.

A limitation of the study is that our data were selfreported via the Internet, which may have introduced measurement error. However, the validity of Internetbased, self-reported anthropometric data is high ${ }^{(43)}$ and has been confirmed in the present study ${ }^{(27)}$. We were not able to include dairy products as a dietary recommendation in the present analyses due to so few individuals meeting the recommendation. However, dairy products do not have a recommended intake in the UK and so habitual diets would not necessarily be expected to comply with this recommendation, even if they were very health conscious. Dietary intakes were estimated by an FFQ, which is known to be subject to misreporting error ${ }^{(44)}$, but this was minimised by validating our FFQ against a $4 \mathrm{~d}$ weighed food record ${ }^{(20)}$. Moreover, our estimation of dietary change was based on the HEI, which is a validated indicator of overall diet ${ }^{(26)}$ and which may be less susceptible to reporting errors than approaches measuring change in specific nutrients or individual foods. Our study participants were almost solely Caucasian; thus, further research in wider ethnicity groups is required to generalise our findings to other populations. One of the primary aims of the Food4Me Proof-of-Principle study was to evaluate change in intakes of food groups across four treatment arms. Thus, although the present study is a secondary analysis of these data, clustering was based on how individuals adhered to food group recommendations and included four clusters. As a result, our analyses are likely to be powered to detect differences between clusters.

\section{Implications of findings}

Our findings suggest that the efficacy of PN in modifying dietary intakes depends on the clustering of adherence to dietary recommendations, with those with the poorest diets benefiting most from the PN intervention. As a result, the implementation of PN-based interventions in individuals with the least healthy diets may help to address health inequalities. Understanding the characteristics of individuals within coherent clusters which are linked with their responsiveness to interventions may help in the design and implementation of more effective health promotion actions. Future PN interventions may benefit from tailoring PN advice based on clustering of overall dietary behaviours rather than on single nutrients or foods.

\section{Conclusions}

We identified four distinct clusters of individuals based on adherence to current food-based dietary recommendations. Participants in the cluster where the fewest recommendations were met (C4) reported significantly greater improvements in their diets (as estimated by the HEI) following a 6-month trial of $\mathrm{PN}$, whereas there was no difference between clusters for those randomised to the Control, non-personalised dietary intervention.

\section{Acknowledgements}

Acknowledgements: The authors would like to thank Antoneta Granic for her valuable input into the design of the cluster analysis. J.A.M. and S.N.-C. are grateful to CIBERobn Fisiopatología de la Obesidad y Nutrición (Instituto Carlos III, Madrid, Spain) for general support in research. Financial support: This work was supported by the European Commission under the Food, Agriculture, Fisheries and Biotechnology Theme of the 7th Framework Programme for Research and Technological Development (265494). The European Commission had no role in the design, analysis or writing of this article. Conflict of interest: None of the authors had a personal or financial conflict of interest. Authorship: K.M.L. and C.C.-M. are joint first authors. Author responsibilities were as follows: Y.M., I.T., C.A.D., E.R.G., L.B., J.A.L., J.A.M., W.H.M.S., H.D., M.G. and J.C.M. contributed to the research design. J.C.M. was the Food4Me Proof-of-Principle study leader. C.C.-M., C.F.M.M., H.F., C.B.O., C.W., A.L.M., R.F., S.N.-C., R.S.-C., S.K., L.T., C.P.L., A.S., M.C.W., E.R.G., L.B. and J.C.M. contributed to the developing the standardised operating procedures for the study. C.C.-M., S.N.-C., R.S.-C., C.W., C.B.O., H.F., C.F.M.M., A.L.M., R.F., S.K., L.T., C.P.L., A.S., M.C.W. and J.C.M. conducted the intervention. C.C.-M., C.F.M.M. and W.H.M.S. contributed to physical activity measurements. K.M.L. and C.C.-M. wrote the paper, performed the statistical analysis for the manuscript. 
All authors contributed to a critical review of the manuscript during the writing process. All authors approved the final version to be published. Ethics of buman subject participation: This study was conducted according to the guidelines laid down in the Declaration of Helsinki and all procedures involving human subjects/patients were approved by the Research Ethics Committees at each University or Research Centre delivering the intervention. The Food4Me trial was registered as an RCT (NCT01530139) at Clinicaltrials.gov. All participants expressing an interest in the study were asked to sign online consent forms at two stages in the screening process. These consent forms were automatically directed to the local study investigators to be counter-signed and archived.

\section{Supplementary material}

To view supplementary material for this article, please visit http://dx.doi.org/10.1017/S1368980016001932

\section{References}

1. Ng M, Fleming T, Robinson M et al. (2014) Global, regional, and national prevalence of overweight and obesity in children and adults during 1980-2013: a systematic analysis for the Global Burden of Disease Study 2013. Lancet 384, 766-781.

2. Hill JO, Wyatt HR \& Peters JC (2012) Energy balance and obesity. Circulation 126, 126-132.

3. NHS Choices (2015) Live Well - Healthy living for everyone. http://www.nhs.uk/Livewell/Pages/Livewellhub.aspx (accessed March 2015).

4. Hood L \& Friend SH (2011) Predictive, personalized, preventive, participatory (P4) cancer medicine. Nat Rev Clin Oncol 8, 184-187.

5. Nielsen DE \& El-Sohemy A (2014) Disclosure of genetic information and change in dietary intake: a randomized controlled trial. PLOS ONE 9, e112665.

6. Berrigan D, Dodd K, Troiano RP et al. (2003) Patterns of health behavior in US adults. Prev Med 36, 615-623.

7. Leech R, McNaughton S \& Timperio A (2014) The clustering of diet, physical activity and sedentary behavior in children and adolescents: a review. Int J Behav Nutr Phys Act 11, 4.

8. Newby PK, Muller D, Hallfrisch J et al. (2003) Dietary patterns and changes in body mass index and waist circumference in adults. Am J Clin Nutr 77, 1417-1425.

9. Moore L, Singer M, Bradlee ML et al. (2015) Adolescent dietary intakes predict cardiometabolic risk clustering. Eur J Nutr 55, 461-468.

10. Khaw K-T, Wareham N, Bingham S et al. (2008) Combined impact of health behaviours and mortality in men and women: the EPIC-Norfolk Prospective Population Study. PLoS Med 5, e12.

11. van Dam RM, Li T, Spiegelman D et al. (2008) Combined impact of lifestyle factors on mortality: prospective cohort study in US women. BMJ 337, a1440.

12. Kesse-Guyot E, Castetbon K, Estaquio C et al. (2009) Association between the French Nutritional Guidelinebased score and 6-year anthropometric changes in a French middle-aged adult cohort. Am J Epidemiol 170, $757-765$.
13. Chiuve SE, Fung TT, Rexrode KM et al. (2011) Adherence to a low-risk, healthy lifestyle and risk of sudden cardiac death among women. JAMA 306, 62-69.

14. Zamora D, Gordon-Larsen P, He K et al. (2011) Are the 2005 dietary guidelines for Americans associated with reduced risk of type 2 diabetes and cardiometabolic risk factors? Twenty-year findings from the CARDIA study. Diabetes Care 34, 1183-1185.

15. Livingstone KM, Celis-Morales C, Navas-Carretero S et al. (2016) Effect of an Internet-based, personalized nutrition randomized trial on dietary changes associated with the Mediterranean diet: the Food4Me Study. Am J Clin Nutr (Epublication ahead of print version).

16. Food4Me (2016) An integrated analysis of opportunities and challenges for personalised nutrition. http://www.food $4 m e$. org/ (accessed February 2016).

17. Celis-Morales C, Livingstone KM, Marsaux CFM et al. (2015) Design and baseline characteristics of the Food4Me study: a web-based randomised controlled trial of personalised nutrition in seven European countries. Genes Nutr 10, 450.

18. Livingstone KM, Celis-Morales C, Navas-Carretero S et al. (2016) Profile of European adults interested in internetbased personalised nutrition: the Food4Me study. Eur J Nutr 55, 759-769.

19. Forster H, Fallaize R, Gallagher C et al. (2014) Online dietary intake estimation: the Food4Me food frequency questionnaire. J Med Internet Res 16, e150.

20. Fallaize R, Forster H, Macready AL et al. (2014) Online dietary intake estimation: reproducibility and validity of the Food4Me food frequency questionnaire against a 4-day weighed food record. J Med Internet Res 16, e190.

21. Food Standards Agency (2002) McCance and Widdowson's The Composition of Foods, sixth summary edition ed. Cambridge: Royal Society of Chemistry.

22. Institute of Medicine (2005) Dietary Reference Intakes for Energy, Carbohydrate, Fibre, Fat, Fatty Acids, Cholesterol, Protein, and Amino Acids. http://www.nap.edu/openbook. php?isbn=0309085373 (accessed March 2015).

23. Institute of Medicine (2011) Dietary Reference Intakes Tables and Applications. http://www.iom.edu/Activities/ Nutrition/SummaryDRIs/DRI-Tables.aspx (accessed March 2015).

24. World Health Organization (2007) Protein and Amino Acid Requirements in Human Nutrition. Report of a Joint WHO/ FAO/UNU Expert Consultation. WHO Technical Report Series no. 935. Geneva: WHO.

25. Food and Agriculture Organization of the United Nations (2010) Fats and fatty acids in human nutrition. Report of an expert consultation. http://www.who.int/nutrition/ publications/nutrientrequirements/fatsandfattyacids_human nutrition/en/ (accessed March 2016).

26. Guenther PM, Casavale KO, Reedy J et al. (2013) Update of the Healthy Eating Index: HEI-2010. J Acad Nutr Diet 113, 569-580.

27. Celis-Morales C, Livingstone KM, Woolhead C et al. (2015) How reliable is internet-based self-reported identity, sociodemographic and obesity measures in European adults? Genes Nutr 73, 476.

28. European Commission (2015) European skills, competences, qualifications and occupations. https://ec.europa. $\mathrm{eu} / \mathrm{esco} / \mathrm{web} /$ guest/hierarchybrowser/-/browser/Occupation (accessed April 2015).

29. European Commission (2015) Mean annual earnings by sex, age and occupation. http://ec.europa.eu/eurostat/web/ products-datasets/-/earn_ses_agt28 (accessed March 2015).

30. Chiu T, Fang D, Chen J et al. (2001) A robust and scalable clustering algorithm for mixed type attributes in large database environment. In Proceedings of the Seventh ACM SIGKDD International Conference on Knowledge Discovery and Data Mining, pp. 263-268. San Francisco, CA: ACM. 
31. Ocké MC (2013) Evaluation of methodologies for assessing the overall diet: dietary quality scores and dietary pattern analysis. Proc Nutr Soc 72, 191-199.

32. Kant AK (2004) Dietary patterns and health outcomes. J Am Diet Assoc 104, 615-635.

33. Ma Y, Bertone ER, Stanek EJ et al. (2003) Association between eating patterns and obesity in a free-living US adult population. Am J Epidemiol 158, 85-92.

34. Wirt A \& Collins CE (2009) Diet quality - what is it and does it matter? Public Health Nutr 12, 2473-2492.

35. US Department of Agriculture (2014) A series of systematic reviews on the relationship between dietary patterns and health outcomes. http://www.nel.gov/vault/2440/web/files/ DietaryPatterns/DPRptFullFinal.pdf (accessed July 2016).

36. Gao SK, Beresford SAA, Frank LL et al. (2008) Modifications to the Healthy Eating Index and its ability to predict obesity: the Multi-Ethnic Study of Atherosclerosis. Am J Clin Nutr $\mathbf{8 8}$, 64-69.

37. Jacobs DR, Sluik D, Rokling-Andersen MH et al. (2009) Association of $1-y$ changes in diet pattern with cardiovascular disease risk factors and adipokines: results from the 1-y randomized Oslo Diet and Exercise Study. Am J Clin Nutr 89, 509-517.
38. Cheng G, Gerlach S, Libuda L et al. (2010) Diet quality in childhood is prospectively associated with the timing of puberty but not with body composition at puberty onset. J Nutr 140, 95-102.

39. Park S-Y, Murphy SP, Wilkens LR et al. (2005) Dietary patterns using the food guide pyramid groups are associated with sociodemographic and lifestyle factors: the Multiethnic Cohort Study. J Nutr 135, 843-849.

40. Di Cesare M, Khang Y-H, Asaria P et al. (2013) Inequalities in non-communicable diseases and effective responses. Lancet 381, 585-597.

41. Celis-Morales C, Lara J \& Mathers JC (2014) Personalising nutritional guidance for more effective behaviour change. Proc Nutr Soc 74, 130-138.

42. Ferguson L (editor) (2013) Nutrigenomics and Nutrigenetics in Functional Foods and Personalized Nutrition. Boca Raton, FL: CRC Press.

43. Pursey K, Burrows LT, Stanwell P et al. (2014) How accurate is web-based self-reported height, weight, and body mass index in young adults? J Med Internet Res 16, e4.

44. Macdiarmid J \& Blundell J (1998) Assessing dietary intake: who, what and why of under-reporting. Nutr Res Rev 11, 231-253. 